OPEN ACCESS

\section{EndoTOFPET-US: a novel multimodal tool for endoscopy and positron emission tomography}

To cite this article: N Aubry et al 2013 JINST 8 C04002

View the article online for updates and enhancements.

\section{Related content}

- EndoTOFPET-US data acquisition system
R Bugalho, C Gaston, M D Rolo et al.
- A 44416 diaital SiPM array with 192
$\frac{\text { TDCs for multiple high-resolution }}{\text { timestamp acquisition }}$
S Mandai and E Charbon
- First characterization of the SPADnet
$\frac{\text { sensor: a digital silicon photomultiplier for }}{\text { PET applications }}$
E Gros-Daillon, L Maingault, L André et al.

\section{Recent citations}

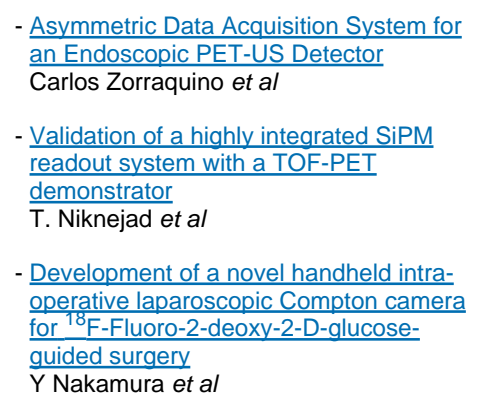


$14^{\text {th }}$ INTERNATIONAL WORKSHOP ON RADIATION IMAGING DETECTORS, 1-5 JULY 2012,

FIGUEIRA DA FOZ, PORTUGAL

\title{
EndoTOFPET-US: a novel multimodal tool for endoscopy and positron emission tomography
}

\author{
N. Aubry, ${ }^{a}$ E. Auffray, ${ }^{b}$ F.B. Mimoun, ${ }^{b}$ N. Brillouet, ${ }^{c}$ R. Bugalho, ${ }^{d}$ E. Charbon, ${ }^{e}$ \\ O. Charles, ${ }^{f}$ D. Cortinovis, ${ }^{g, h}$ P. Courday ${ }^{c}$ A. Cserkaszky, ${ }^{i}$ C. Damon, ${ }^{f}$ K. Doroud,${ }^{b}$
} J.-M. Fischer, ${ }^{i}$ G. Fornaro, ${ }^{b}$ J.-M. Fourmigue, ${ }^{a}$ B. Frisch, ${ }^{b}$ B. Fürst, ${ }^{j}$ J. Gardiazabal, ${ }^{j}$ K. Gadow, ${ }^{g}$ E. Garutti, ${ }^{h}$ C. Gaston, ${ }^{d}$ A. Gil-Ortiz, ${ }^{k}$ E. Guedj, ${ }^{f}$ T. Harion, ${ }^{k}$ P. Jarron, ${ }^{b}$ J. Kabadanian, ${ }^{f}$ T. Lasser, ${ }^{j}$ R. Laugier, ${ }^{f}$ P. Lecoq,${ }^{b}$ D. Lombardo, ${ }^{l}$ S. Mandai, ${ }^{e}$ E. Mas, ${ }^{l}$ T. Meyer, ${ }^{b}$ O. Mundler,${ }^{f}$ N. Navab, ${ }^{j}$ C. Ortigão, ${ }^{d}$ M. Paganoni, ${ }^{m}$ D. Perrodin, ${ }^{a}$ M. Pizzichemi, ${ }^{m}$ J.O. Prior, ${ }^{n}$ T. Reichl, ${ }^{j}$ M. Reinecke, ${ }^{g}$ M. Rolo, ${ }^{d}$ H.-C. Schultz-Coulon, ${ }^{k}$ M. Schwaiger, ${ }^{o}$ W. Shen, ${ }^{k}$ A. Silenzi, ${ }^{g}, 1$ J.C. Silva, ${ }^{d}$ R. Silva, ${ }^{d}$ I. Somlai Schweiger ${ }^{o}$ R. Stamen, ${ }^{k}$ J. Traub, ${ }^{i}$ J. Varela, ${ }^{d}$ V. Veckalnss, ${ }^{d}$ V. Vidal, ${ }^{f}$ J. Vishwas, ${ }^{e}$ T. Wendler, ${ }^{i}$ C. Xu, ${ }^{g, h}$ S. Ziegler ${ }^{o}$ and M. Zvolsky ${ }^{g, h}$

\footnotetext{
${ }^{a}$ Fibercryst SAS, La Doua - Bat. l'Atrium Bd LATARJET,

F-69616 Villeurbanne, France

${ }^{b}$ CERN, European Organization for Nuclear Research, CH-1211 Geneva 23, Switzerland

${ }^{c}$ Kloe SA, Hotel d'Entreprise du Millenaire, 1068 Rue de la Vieille, Poste 34000, Montpellier, France

${ }^{d}$ Laboratório de Instrumentacão e Física Experimental de Partículas, Av. Elias Garcia 14-1º, 1000-149 Lisboa,Portugal

${ }^{e}$ Delft Technical University,

Stevinweg 1, 2628 CN Delft, Netherlands

${ }^{f}$ Université de la Méditeranée, Aix-Marseille II, $7^{\mathrm{me}}$ arrondissement, F-13007 Marseille, France

${ }^{g}$ Deutsches Elektronen-Synchrotron DESY, Notkestrasse 85, D-22607, Hamburg, Germany

${ }^{h}$ Universty of Hamburg, Department of Physics, Jungiustrasse 9, D-20355 Hamburg, Germany
}

\footnotetext{
${ }^{1}$ Corresponding author.
} 


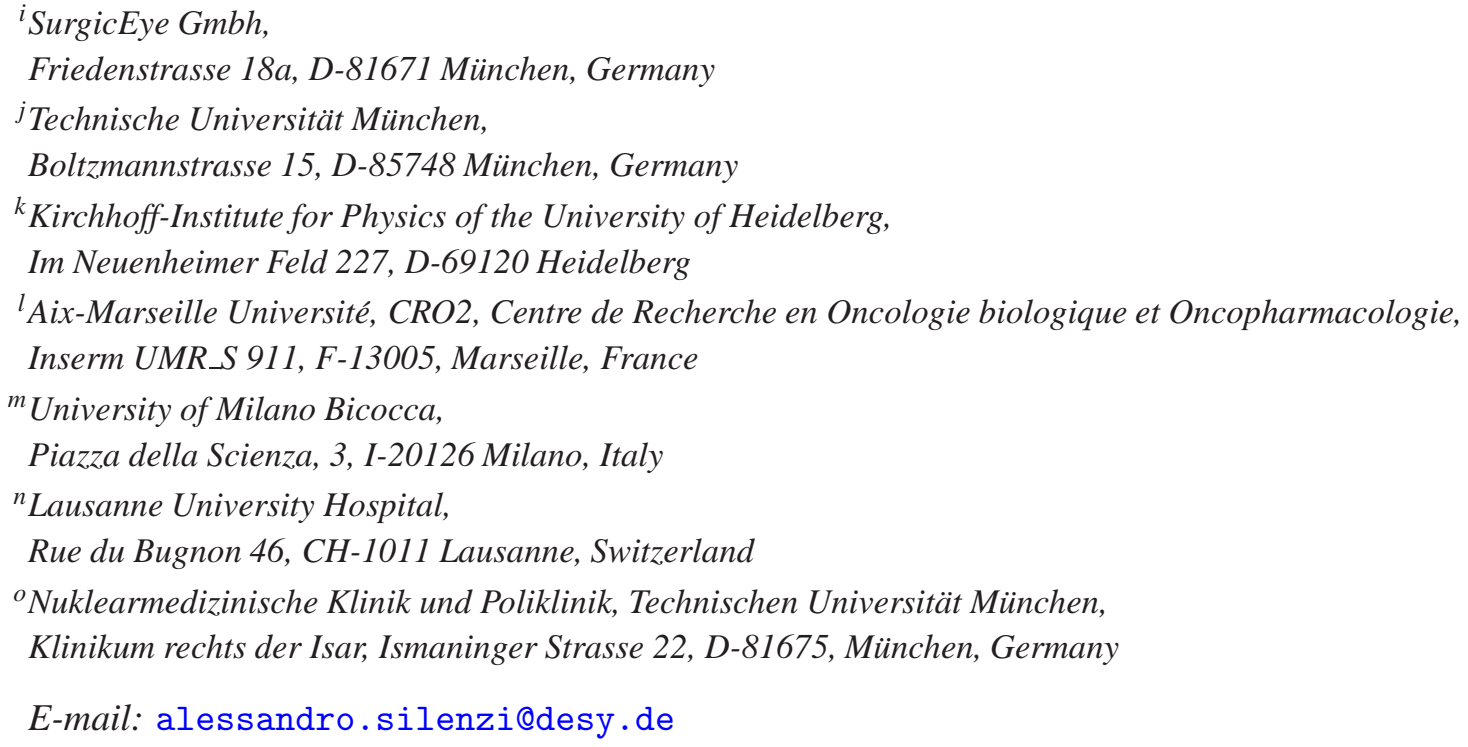

ABSTRACT: The EndoTOFPET-US project aims to develop a multimodal detector to foster the development of new biomarkers for prostate and pancreatic tumors. The detector will consist of two main components: an external plate, and a PET extension to an endoscopic ultrasound probe. The external plate is an array of LYSO crystals read out by silicon photomultipliers (SiPM) coupled to an Application Specific Integrated Circuit (ASIC). The internal probe will be an highly integrated and miniaturized detector made of LYSO crystals read out by a fully digital SiPM featuring photosensor elements and digital readout in the same chip. The position and orientation of the two detectors will be tracked with respect to the patient to allow the fusion of the metabolic image from the PET and the anatomic image from the ultrasound probe in the time frame of the medical procedure. The fused information can guide further interventions of the organ, such as biopsy or in vivo confocal microscopy.

KEYWORDs: Gamma camera, SPECT, PET PET/CT, coronary CT angiography (CTA); Multimodality systems; Intra-operative probes 


\section{Contents}

1 The EndoTOFPET-US and its clinical case 1

2 Technological specifications $\quad 1$

2.1 External plate 3

2.2 Internal probe 3

3 Status and outlook 5

\section{The EndoTOFPET-US and its clinical case}

The EndoTOFPET-US project aims to jointly exploit Time-Of-Flight Positron Emission Tomography (TOFPET) and ultrasound (US) endoscopy with a multimodal instrument for diagnostic and therapeutic oncology [1]. The clinical cases targeted by this project are prostate and pancreas tumors. Both organs are commonly examined using endoscopic ultrasound procedures through natural orifices and could benefit of the molecular information of PET images. It is important however to consider that are surrounded by organs with high uptake in PET such as the heart, the liver and the blood pool for the pancreatic case or the bladder for the case of the prostate [2]. In these cases, the selection of the Region Of Interest (ROI) using TOF is crucial to the quality of the positron emission image in order to reduce the background from the neighboring organs. The present technological and physiological limitations will be addressed through the development of this multimodal device.

\section{Technological specifications}

The detector consists of two main components: a PET head extension for a commercial ultrasound endoscope, depicted in figure 1, and an outer PET plate, shown in figure 2 facing the inner probe. The size of the pancreatic endoscope and its PET extension is about half that of the prostate one. The in vivo configuration is sketched in figure 3 . The project aims to develop a detector unit, which fits the prostate endoscope needs and can be scaled for the adaptation to the pancreatic endoscope. For time and economic reasons only one of the two detectors will be produced and commissioned for clinical applications. The prostate detector has been chosen as the simplest integration task, but the design will maintain all the requirements of scalability for the pancreas solution.

The position along the line of response (LOR) in a TOF-PET can be calculated with the formula $t_{1}-t_{2}=\left(x_{1}-\left(L-x_{1}\right)\right) / c$, where $x_{1}$ is the distance from detector $1, L$ is the length of the LOR, $t_{1}$ and $t_{2}$ is the time recorded by detectors 1 and 2. Consequentially coincidence time-offlight resolution (CTR) corresponds to a spatial resolution $\Delta x=c \cdot \Delta t / 2$. The average healthy prostate is an irregularly shaped organ of roughly $4 \times 3 \times 2 \mathrm{~cm}^{3}$ [2], so a CTR of 200 ps Full Width Half Maximum (FWHM) (equivalent to $3 \mathrm{~cm}$ along the line of response) is mandatory to suppress 


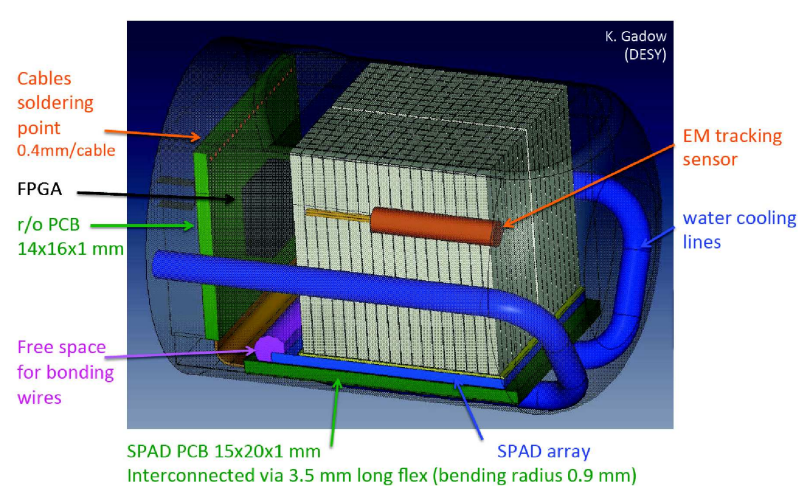

Figure 1. 3D drawing of the EndoTOFPET-US prostate probe. The device has a diameter of $23 \mathrm{~mm}$ and will be sealed in a casing and mounted on an Hitachi transrectal ultrasound probe. The picture highlights the parts of the detector head, such as crystal matrices, the digital SiPM chip (SPAD array) and connections to DAQ board, the magnetic tracking sensor, and a $1 \mathrm{~mm}$ diameter pipe for cooling water

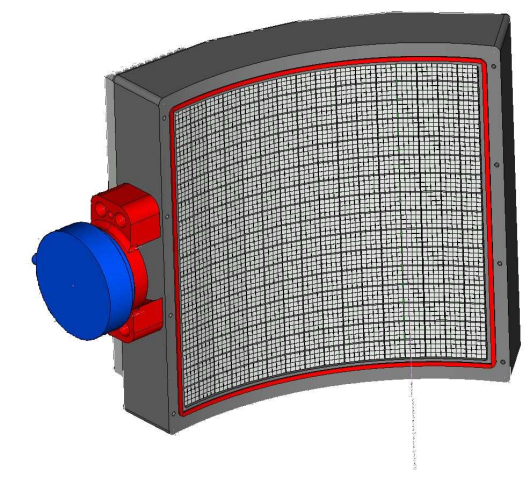

Figure 2. 3D drawing of the EndoTOFPET-US external plate. The picture shows the crystal matrices arranged in a pointing geometry, the aluminium casing and the attachment for a robotic arm.

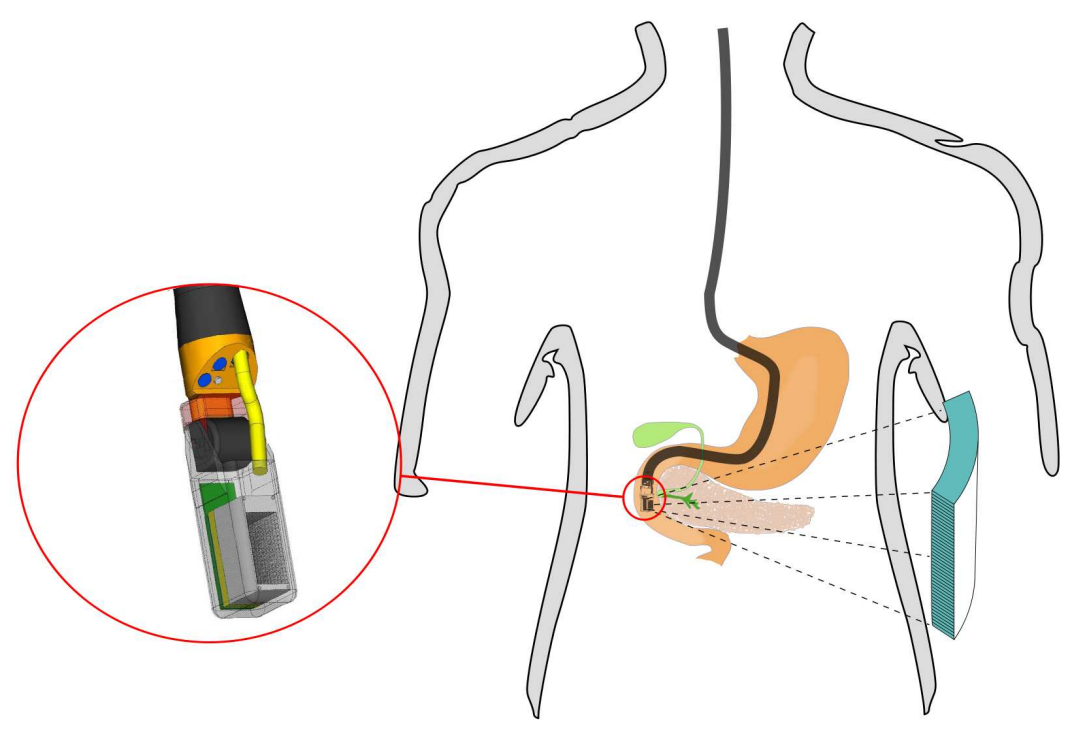

Figure 3. Sketch of the in vivo configuration for the pancreatic clinical case. Left is depicted the endoscope PET extension for the pancreatic clinical case, positioned under the bend of the duodenum opposite to the external plate, enclosing the pancreas in the field of view.

the background from organs surrounding the ROI. First measurements on LSO crystal show a direct dependence of the CTR from the crystal length [3]. However beneficial for the CTR, short crystals $(5 \mathrm{~mm})$ cannot be employed in this design at the expense of the sensitivity of the system. Simulation studies indicate that the best trade off between sensitivity and time resolution is obtained with crystals of $[10,15] \mathrm{mm}$ length [4]. The simulation predicts a CTR of $180 \pm 5 \mathrm{ps}$ for $10 \mathrm{~mm}$ LYSO crystals and $200 \pm 5$ ps for $15 \mathrm{~mm}$. The deterioration of the time resolution of a factor $10 \%$ is paid off by the increase in sensitivity of a factor $30 \%$ in favor of the $15 \mathrm{~mm}$ case. The design foresees 
$15 \mathrm{~mm}$ crystals for the external plate and $10 \mathrm{~mm}$ crystals for the internal probe. The latter choice is constrained by the mechanical requirements for the housing the internal probe.

\subsection{External plate}

The external plate is a detector of $23 \times 23 \mathrm{~cm}^{2}$ composed of 256 elements. An element consists of a $4 \times 4$ array of $3 \times 3 \times 15 \mathrm{~mm}^{3}$ LYSO crystals individually coupled to a monolithic matrix of $4 \times 4$ Multi-Pixel Photon Counter (MPPC), each one with an active area of $3 \times 3 \mathrm{~mm}^{2}$ from Hamamatsu [5]. The MPPC is soldered to a printed circuit board (PCB) that incorporates a low pass filter for the bias voltage of the MPPC, a temperature sensor (Dallas DS18B20U), and a connector. The footprint of the PCB of the detector element is smaller than the package of the MPPC, this solution reduces the gap between the adjacent elements to the minimum. The design of the external plate is shown in figure 2. The signals from the detector elements are transferred through a flexible printed circuit to the Front End Boards (FEB). The FEB hosts the ASICs which digitize the MPPC signals, containing the energy deposited and time-of-arrival (TOA). The ASIC features a self triggering mechanism based on a double threshold system that will be able to record the time of arrival of the first photoelectron, while rejecting the frequent dark counts from the MPPC (up to $2 \times 10^{6} \mathrm{~s}^{-1}$ per channel). The data from the FEB is delivered to the DAQ server in conjunction with the position of the detectors, which is measured with optical and magnetic systems with accuracy of $0.3 \mathrm{~mm}$ RMS and $0.6 \mathrm{~mm}$ RMS respectively. The position of the external plate is determined by a robotic arm that holds the structure and is automatically positioned to optimize the field of view depending on the orientation of the internal probe. In the current development status, $3 \times 3 \times 15 \mathrm{~mm}^{3}$ LYSO:Ce crystals have been coupled to a MPPC S10931-050P and a NINO differential amplifier-discriminator [3], yielding an average time resolution of $235 \pm 4$ ps. The MPPC matrix has been tested extensively showing a performance similar to a single MPPC. A minor deterioration of the time resolution is observed in the monolithic matrix with respect to the single MPPC. This effect is currently under investigation and the full readout chain will be characterized in a multi-channel system.

\subsection{Internal probe}

The internal probe designed for the prostate case is composed of a commercial transrectal ultrasound probe (Hitachi medical systems EUP-U533) and by a highly integrated extension shown in figure 1. The extension has the same cross-section of the ultrasound probe and hosts a matrix of $18 \times 18$ LYSO crystals of $0.71 \times 0.71 \times 10 \mathrm{~mm}^{3}$. Each crystal is individually coupled to a digital silicon photomultiplier (dSiPM) comprising a cluster of 416 single photon avalanche diodes (SPAD). A SPAD is a Geiger mode avalanche photodiode fabricated in a standard CMOS process [6], the most employed technology to be implemented in very large scale integration chips. This technological choice allows the integration of individual pixel digital readout and multiple time-to-digital-converters (TDC) on the same chip that hosts the photosensors. The chip acts as an array of fully digital SiPMs, with the practical possibility of incorporating one TDC per SPAD and measuring the TOA of each scintillation photon detected by the digital SiPM. However this solution would reduce the fill factor and consequently the effective light yield and worsen the CTR $[4,7]$. The trade-off envisaged for the digital SiPM is to maximize the fill factor by reducing the digital elements per pixel to the minimum and sharing a bank of TDC between columns of pixels, with up 


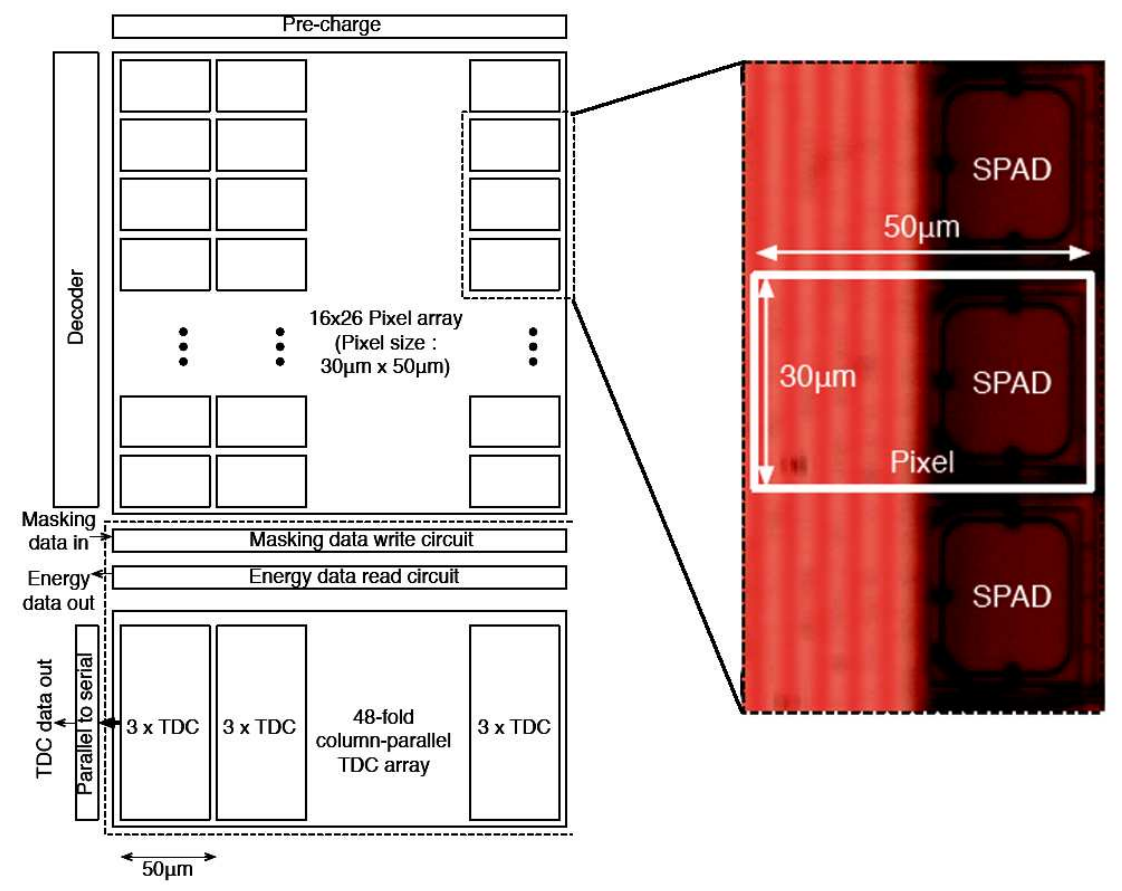

Figure 4. Digital SiPM in CMOS technology. The picture shows on the left the block diagram of the architecture and on the right a micrographic detail of the pixel element, the vertical lines are the connection lines column-wise readout of the SPAD pixels, the active area is indicated with the label SPAD.
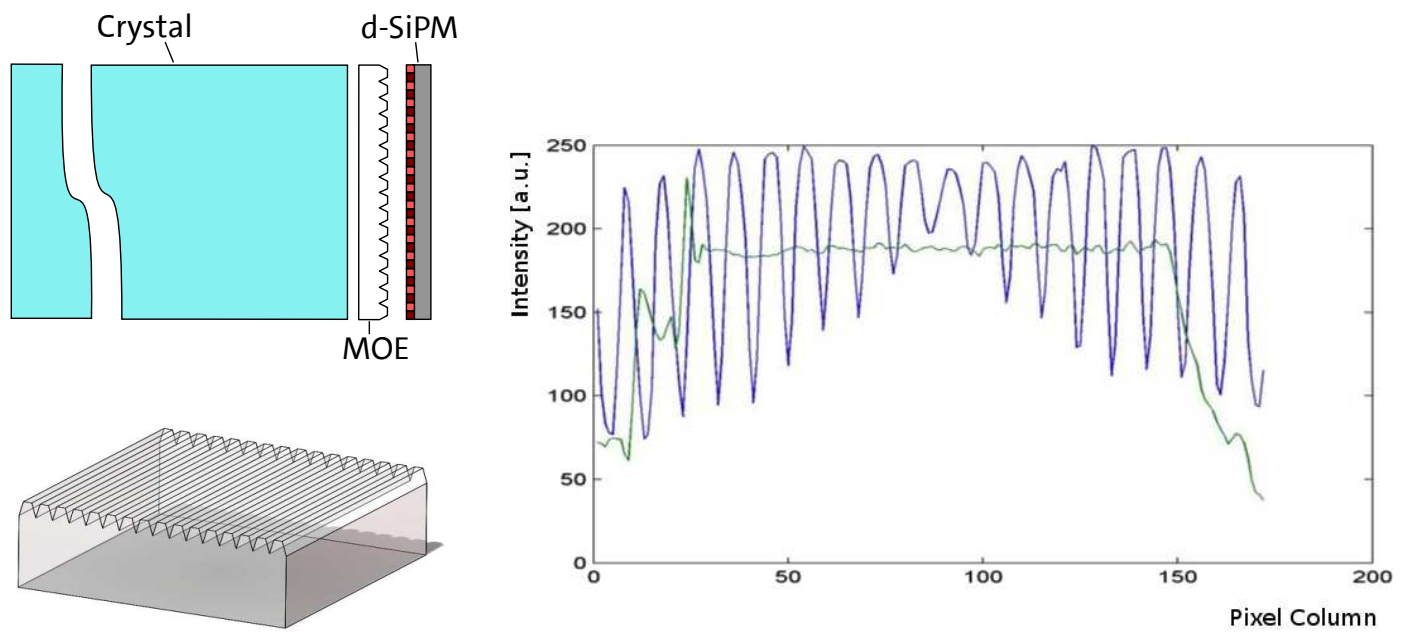

Figure 5. Top: Sketch of the assembly of crystal, microoptical element (MOE) and digital SiPM. Bottom: Depiction of Figure 6. light distribution of an X-ray excited LYSO crystal measured with a CCD camera with (blue) and without (green) optical elements. the micro optical element structure. 
to 48 time measurements per crystal each event. Figure 4 shows the block diagram of one cluster of the digital SiPM and the micrograph of the SPADs. The geometric fill factor will be enhanced by exploiting the regular structure of the active elements using a micro optical grating to redirect the scintillation light from the insensitive areas to the SPAD.

Figure 5 depicts of the micro optical elements and the assembly of them between crystal and digital SiPM. In order to measure the performances of the micro optical elements (MOE), a beam of X-rays $(40 \mathrm{keV})$ is directed to the side of the crystal matrix wrapped in Vikuiti ${ }^{\mathrm{TM} 1}$ and the scintillation light is measured by a photosensor. Figure 6 shows the distribution of the scintillation light from the first row of crystals, fully absorbing the beam due to the high absorption coefficient for soft X-rays. The light distribution is measured using a high resolution CCD sensor attached to the face of the crystal for the naked crystal (green) and the crystal with the MOE (blue). The net average light yield is $26 \%$ for the sensitive areas. A detailed GEANT4 simulation of the behavior of the digital SiPM [4] has been configured using the values of dark count rate, photodetection efficiency (PDE), single pixel time resolution (SPTR) and temperature dependence measured from prototype models of the digital SiPM. The dSiPM is simulated attached to a LYSO crystal of $0.71 \times 0.71 \times 10 \mathrm{~mm}^{3}$ with a light output of $32 \mathrm{kPh} / \mathrm{MeV}$, rise time of $100 \mathrm{ps}$ and decay time of $40 \mathrm{~ns}$. The results of the simulation guided the selection process of the cluster architecture for the final production. The simulation predicts a CTR of $184 \mathrm{ps}$ and a further improvement of $[10,15] \%$ when the effective PDE is enhanced using the micro optical elements. First tests have been performed with $0.71 \times 0.71 \times 10 \mathrm{~mm}^{3}$ LYSO:Ce crystals coupled to a MPPC S10931050P and a NINO differential amplifier-discriminator [3], showing an average time resolution of $187 \pm 27 \mathrm{ps}$.

\section{Status and outlook}

A preliminary design has been developed for the external plate and the prostate option of the extension of the ultrasound probe. Time resolution studies performed with crystals and MPPC for the external plate and the internal probe can be combined to a CTR of $212 \pm 22 \mathrm{ps,} \mathrm{which} \mathrm{is} \mathrm{consistent}$ with the goal of $200 \mathrm{ps}$ of the project. The next step toward the complete system is to repeat or improve these measurements with the $\mathrm{dSiPM}$ and the ASIC. The result obtained for the individual components are very promising and show that the EndoTOFPET-US project is on schedule towards the manufacturing of the complete system by the end of next year.

\section{Acknowledgments}

The author wishes to thank the collaboration for the privilege of speaking on their behalf in presenting these preliminary results and design. The research leading to these results has received funding from the European Union Seventh Framework Program [FP7/2007-2013] under Grant Agreement $\mathrm{n}^{\circ} 256984$.

\footnotetext{
${ }^{1}$ Enhanced specular reflector foil produced by $3 \mathrm{M}$ company.
} 


\section{References}

[1] EndoTOFPET-US Proposal: Novel multimodal endoscopic probes for simultaneous PET/ultrasound imaging for image-guided interventions, European Union $7^{\text {th }}$ Framework Program (FP7/2007-2013) under Grant Agreement No. 256984, Health-2010.1.2-1.

[2] S. Stranding, Gray's anatomy, 40 ${ }^{\text {th }}$ edition, pp. 1125, 1126, 1245, Elsevier.

[3] E. Auffray et al., A Comprehensive and Systematic Study of Coincidence Time Resolution and Light Yield Using Scintillators of Different Size, Wrapping and Doping, in the proceedings of IEEE Nucl. Sci. Symp. MIC (2011).

[4] E. Garutti et al., Single Channel Optimization for an Endoscopic Time-of-Flight Positron Emission Tomography Detector, in the proceedings of IEEE Nucl. Sci. Symp. MIC (2011).

[5] Hamamatsu Solid state division, Monolithic MPPC array in SMD package S11828-3344M manual, available online http://www.hamamatsu.com.

[6] S. Mandai, V. Jain and E. Charbon, A Fully-Integrated $780 \times 800 \mu \mathrm{m}^{2}$ Multi-Digital Silicon Photomultiplier With Column-parallel Time-to-Digital Converter, in the proceedings of ESSIRC 2011.

[7] S. Seifert, HT. van Dam and D.R. Schaart, The lower bound on the timing resolution of scintillation detectors, Phys. Med. Biol. 57 (2012) 1797. 